\title{
ASPECTOS TÉCNICOS E RELACIONAIS DA INTERCONSULTA PSICOLÓGICA: A VISÃO DOS PSICÓLOGOS
}

Thaís de Castro Gazotti

PUC-Campinas

\author{
Helena Bazanelli Prebianchi \\ PUC-Campinas
}

\begin{abstract}
Resumo
A interconsulta psicológica é uma das atividades mais frequentes do psicólogo hospitalar. Estudos reconhecem sua importância em favorecer as relações entre a equipe de saúde e o paciente, seus familiares e seus profissionais. Este estudo objetivou conhecer os aspectos metodológicos e técnicos do serviço de interconsulta psicológica de um Hospital Universitário, a partir das vivências dos profissionais que o efetivam. Realizaram-se entrevistas com quatro psicólogas, sendo duas residentes e duas assistenciais contratadas, as quais foram gravadas em áudio, transcritas e analisadas de acordo com o método de análise de conteúdo de Bardin. Os resultados indicaram a importância da interconsulta psicológica como atividade interdisciplinar, mas que a mesma ocorre sem qualquer sistematização, dificultando a avaliação dos seus efeitos e replicabilidade. Concluiu-se pela necessidade de procedimentos padronizados, atendimento a protocolos de intervenção e novos estudos sobre o tema.
\end{abstract}

Palavras-chave: psicologia; hospitais gerais; intervenção psicológica; pacientes; interconsulta médico-psicológica.

\section{PSYCHOLOGICAL CONSULTATION-LIAISON: METHODOLOGICAL AND TECHNICAL ASPECTS}

\begin{abstract}
The psychological consultation-liaison is one of the most frequent activities performed by a hospital psychologist. Several studies recognized its importance in supporting the relationship between the healthcare team and patients, relatives and among professionals. This study aimed to identify the methodological and technical aspects of the psychological consultation-liaison service in a university hospital considering the professionals lived experiences. Four psychologists were interviewed in this study, two interns and two professionals. The interviews were audio-recorded, transcribed and analyzed according to Bardin's content analysis method. The results indicated the importance of psychological consultation-liaison as an interdisciplinary activity but it occurs without any systematization hindering the evaluation of its effects and replicability. It was concluded for the need of standardized procedures, attention to protocols of intervention and new studies on this subject.
\end{abstract}

Keywords: psychology; hospital general; psychological intervention; patients; physician-psychological consultation liaison. 


\title{
CONSULTA PSICOLÓGICA DE ENLACE: ASPECTOS METODOLÓGICOS Y TÉCNICOS
}

\begin{abstract}
Resumen
La interconsulta psicológica es una de las actividades más frecuentes del psicólogo hospitalario. Varios estudios reconocen la importancia de favorecer las relaciones entre el equipo de salud y e el paciente, sus familiares y sus profesionales. Se objetivo conocer los aspectos metodológicos y técnicos del servicio de interconsulta psicológica de un hospital universitario, a partir de las vivencias de los profesionales psicólogos que lo realizan. Fueron realizadas entrevistas con cuatro psicólogas, dos residentes y dos contratistas, que fueron grabadas en audio, transcriptas y analizadas en consonancia con el método de análisis de contenido de Bardin. Los resultados indicaron la importancia de la interconsulta psicológica como actividad interdisciplinar, pero la misma ocurre sin cualquier sistematización, dificultando la evaluación de sus efectos y replicabilidad. Se concluyó por la necesidad de procedimientos estandarizados, atención a los protocolos de intervención y nuevos estudios sobre el tema.

Palabras clave: psicología; hospitales generales; intervención psicológica; pacientes; interconsulta medico-psicológica.
\end{abstract}

\section{INTRODUÇÃO}

A atuação do psicólogo ampliou-se por volta da década de 1960 ao deixar seu campo de prática, até então restrito a consultório particular e instituição psiquiátrica, para atuar em um novo contexto: os hospitais gerais, que proporcionaram o desenvolvimento de um campo de atuação específico, no Brasil, denominado Psicologia Hospitalar (Melo, 2015). No entanto, a terminologia Psicologia Hospitalar não se adequada, pois restringe a um local a prática profissional sendo o termo mais correto Psicologia da Saúde, assim, a Psicologia no hospital seria compreendida como uma sub-especialidade (Azevêdo \& Crepaldi, 2016).

A interconsulta, criada nos hospitais gerais a partir da entrada da psiquiatria nesse contexto (De Marco, 2003; citado por Rossi, 2008), passou a ser realizada também pelos psicólogos quando estes se inseriram no ambiente hospitalar (Rossi, 2008). A interconsulta consiste na presença de um profissional de saúde em uma unidade ou serviço médico geral atendendo à solicitação de um médico em relação ao atendimento de um paciente (Carvalho \& Lustosa, 2008). Em linhas gerais, segundo Andreoli e Mari (2002), a interconsulta caracteriza-se por assistir a quem dá assistência, cooperando na tarefa a ser realizada e trabalhando em conjunto com a equipe.

De modo específico, a interconsulta psicológica se caracteriza pelo diagnóstico e tratamento de problemas psicológicos, das dificuldades interpessoais e dos dilemas institucionais que envolvam o paciente, a família e a equipe de saúde, facilitando a comunicação, a cooperação e a elaboração dos conflitos dos envolvidos (Santos, Messias, Prebianchi, Oliveira, \& Cardoso, 2011). Em outras palavras, nesta atividade, cabe ao psicólogo a tarefa da escuta, do acolhimento e da compreensão dos aspectos psíquicos e psicossociais do ser humano com o objetivo de prestar assistência ao paciente, de modo a contribuir para uma melhor 
relação profissional-paciente e um ambiente humanizado (Toro, Nucci, Toledo, Oliveira, \& Prebianchi, 2013).

De acordo com Azevêdo e Crepaldi (2016), a presença do psicólogo no contexto hospitalar em diálogo constante com os demais profissionais da equipe multidisciplinar concretiza a perspectiva interdisciplinar do cuidado. A interdisciplinaridade possibilita que haja uma aproximação entre os profissionais da saúde - suas práticas e áreas de saberes - de modo a contextualizar as ações em saúde diante de sua complexidade e extensão (Giacomozzi, 2012).

Deste modo, graças a sua natureza interdisciplinar, a interconsulta psicológica tem sido reconhecida como uma das intervenções mais visíveis do psicólogo hospitalar frente aos demais profissionais da equipe, principalmente nos hospitais universitários (Gazotti \& Prebianchi, 2014; Rossi, 2008). Em estudo realizado por Gazotti e Prebianchi (2014), constatou-se que, na maioria das vezes, a interconsulta é solicitada principalmente por médicos e enfermeiros, quando estes identificam os pacientes como apresentando humor depressivo, ansiedade diante do diagnóstico ou pelo longo período de internação.

Trabalhos como os de Nogueira-Martins (1995) e Rossi (2008) referem que, em termos de processo, o interconsultor realiza, primeiramente, uma análise funcional do ambiente e das demandas impostas por pacientes e equipe de saúde, por meio de coleta de informações junto a estes e seus familiares. O diagnóstico situacional envolve a explicitação de reações psicológicas de todos os envolvidos naquela situação, inclusive as reações do interconsultor. Em seguida, é realizada a devolução ao solicitante das avaliações sobre o funcionamento do paciente, família e equipe, as inter-relações contextualizadas no ambiente hospitalar e as estratégias de intervenção possíveis.

Contudo, na prática, a irregularidade das solicitações de interconsulta psicológica por outros profissionais da equipe, a formação falha dos psicólogos, a não padronização da avaliação e dos critérios para diagnóstico e a falta de produções de evidências que ressaltem a eficácia das intervenções efetuadas, têm contribuído para a desvalorização deste campo de atuação profissional (Andreoli \& Mari, 2002: Carvalho \& Lustosa, 2008). Também ressalta-se que, para que haja uma boa inserção e adequação do psicólogo à equipe multiprofissional, é essencial que os demais profissionais compreendam as funções do mesmo (Gazotti, 2017).

Dessa forma, a inserção reconhecida do psicólogo na equipe multiprofissional, através da atividade de interconsulta, requer a qualificação desta prática profissional. Neste sentido, conhecer e compreender os aspectos processuais, metodológicos e técnicos envolvidos é o primeiro passo para a avaliação e aperfeiçoamento da prestação desse serviço aos usuários internos (equipe multiprofissional) e externos (pacientes) ao hospital geral. Por isso, o presente estudo teve como objetivo conhecer, a partir das vivências dos profissionais psicólogos, os aspectos metodológicos e técnicos da interconsulta psicológica realizada em um hospital universitário. 


\section{MÉTODO}

Consonante com o objetivo do estudo, procedeu-se uma pesquisa qualitativa, envolvendo os profissionais que realizavam interconsulta psicológica no hospital.

\section{Contexto}

A pesquisa foi realizada num hospital universitário, caracterizado por ser uma das principais instituições hospitalares de atuação terciária de uma cidade no interior paulista e sua região metropolitana. Atualmente, conta com 353 leitos ativos, sendo 243 destinados exclusivamente ao convênio do Sistema Único de Saúde (SUS). Participa no atendimento à população com uma média mensal de 20 mil consultas ambulatoriais, 15 mil atendimentos nas Unidades de Urgência e Emergência, 1.250 procedimentos cirúrgicos, além de 1.600 internações.

Quanto ao Serviço de Psicologia (atuando desde 1990), o hospital universitário possuía, por ocasião do estudo, duas psicólogas assistenciais contratadas, duas psicólogas do Programa de Residência em Psicologia da Saúde/Hospitalar e três psicólogas do Programa de Aprimoramento Profissional em Psicologia da Saúde/Hospitalar. Em relação às interconsultas psicológicas, ocorrem, em média, 300 por ano.

\section{Participantes}

Participaram do estudo quatro psicólogas; duas psicólogas residentes (nomeadas de P1 e P2) e duas assistenciais contratadas (nomeadas de P3 e P4), que atendiam ao critério de inclusão, isto é, realizar interconsultas. Os demais membros do Serviço de Psicologia não foram incluídos, pois não atendiam ao referido critério.

\section{Instrumentos}

Trata-se de uma pesquisa qualitativa, a qual utilizou para coleta de dados a entrevista semiestruturada que, inicialmente, incluía itens relativos à identificação dos participantes. A segunda parte da entrevista foi composta por questões formuladas ao considerar os objetivos da pesquisa. As questões visavam explorar os seguintes aspectos: como as psicólogas atendem às solicitações de interconsulta psicológica; com quem e como é feito o primeiro contato; quais as estratégias e aspectos abordados no atendimento psicológico ao paciente durante a interconsulta; como é feita a devolutiva do atendimento psicológico ao solicitante da interconsulta; como é realizada a interconsulta psicológica quando há presença de acompanhante do paciente e, finalmente, quais as dificuldades encontradas e os aspectos a serem cuidados para o bom funcionamento do Serviço de Interconsulta Psicológica. 


\section{Procedimentos}

Após a aprovação do projeto pelo Comitê de Ética em Pesquisa com Seres Humanos da universidade onde foi realizada a pesquisa, sob protocolo 0598/11, as entrevistas foram realizadas por uma das pesquisadoras após um estudo piloto, no qual foi utilizado o roteiro de questões previamente elaborado. Diante dos resultados satisfatórios, prosseguiu-se com a realização das demais entrevistas.

As entrevistas foram previamente agendadas com as participantes e ocorreram em uma sala de arquivos nas dependências do Hospital Universitário, com duração média de 40 minutos cada uma. No início de cada um dos encontros, a pesquisadora apresentou à participante o Termo de Consentimento Livre e Esclarecido para ser lido e assinado. Em seguida, iniciou a entrevista a partir dos aspectos definidos no roteiro.

Todas as entrevistas foram áudio gravadas e, posteriormente, transcritas. Os dados coletados foram analisados de acordo com a técnica de análise de conteúdo do tipo categorial temática de Bardin (1988).

\section{RESULTADOS}

Quanto às características sócio demográficas dos participantes, pode-se observar os dados apresentados na Tabela 1.

Tabela 1. Características sócio demográficas dos Participantes

\begin{tabular}{|c|c|c|c|c|}
\hline Participantes & Idade & $\begin{array}{c}\text { Instituição de } \\
\text { Formação/Estado }\end{array}$ & $\begin{array}{c}\text { Período de } \\
\text { Atuação no } \\
\text { Hospital }\end{array}$ & $\begin{array}{c}\text { Posição } \\
\text { Profissional }\end{array}$ \\
\hline $\mathrm{P} 1$ & 27 anos & $\begin{array}{l}\text { Universidade } \\
\text { Particular/SP }\end{array}$ & $\begin{array}{c}1 \text { ano e } 9 \\
\text { meses }\end{array}$ & Residente 2 \\
\hline P2 & 25 anos & $\begin{array}{l}\text { Universidade } \\
\text { Federal/MG }\end{array}$ & $\begin{array}{c}1 \text { ano e } 9 \\
\text { meses }\end{array}$ & Residente 2 \\
\hline P3 & 34 anos & $\begin{array}{l}\text { Universidade } \\
\text { Particular/SP }\end{array}$ & $\begin{array}{c}2 \text { anos } \\
\text { (residência) e } \\
4 \text { meses }\end{array}$ & Assistencial \\
\hline P4 & 33 anos & $\begin{array}{l}\text { Universidade } \\
\text { Particular/SP }\end{array}$ & quase 8 anos & Assistencial \\
\hline
\end{tabular}

Os resultados mostraram que três participantes (P2, P3 e P4) eram graduadas em universidades particulares do Estado de São Paulo e uma (P1) em universidade federal do Estado de Minas Gerais. As idades das participantes variavam de 25 a 35 anos: psicólogas residentes (P1 e P2) encontravam-se na faixa etária de 25 e 30 anos e as psicólogas assistenciais (P3 e P4) encontravamse entre 30 e 35 anos de idade.

As psicólogas residentes atuavam exclusivamente no hospital, aproximadamente há dois anos. As psicólogas assistenciais possuíam outras 
atuações, tais como docência em universidade e consultório particular, presentes na área hospitalar há 3 e 8 anos, respectivamente.

Em relação às entrevistas realizadas, foram identificadas seis categorias temáticas a partir do tema interconsulta, conforme apresentado na Tabela 2. No relato de todas participantes, foram abordadas cada uma das categorias temáticas revelas pela análise.

Tabela 2. Categorias Temáticas abordadas no estudo Tema

Categoria Temática
A demanda por interconsulta
O primeiro contato
$\mathrm{O}$ atendimento ao paciente

Interconsulta

O encerramento junto ao paciente

Finalização do processo de interconsulta psicológica

junto ao solicitante

Limitações e vicissitudes da prática

A seguir são apresentados os trechos dos depoimentos das participantes que ilustram suas vivências em relação à interconsulta.

\section{A demanda por interconsulta}

Os relatos das entrevistadas indicaram que as solicitações de interconsulta psicológica ocorrem de duas maneiras. Uma delas é caracterizada por ser formal, ou seja, por meio de um Formulário de Solicitação de Interconsulta padronizado pelo Hospital, a outra maneira e mais frequente, é informal que ocorre mediante contato pessoal ou telefônico particular.

\footnotetext{
"Na residência eu era informada a partir da solicitação de interconsulta oficial, que é aquela folha amarelinha, e, também, por comunicação da equipe - alguém me procurava e verbalmente me avisava (...). $E$, alguns momentos, por telefone, também, alguns profissionais entravam em contato." (P3).
}

\section{O primeiro contato}

Pelas normas hospitalares, após receber uma solicitação de interconsulta psicológica, as psicólogas têm 48 horas para atender ao pedido. De acordo com os depoimentos das mesmas, como primeiro contato procuram conversar com o profissional solicitante para, então, ir ao encontro do paciente. Contudo, devido à alta rotatividade do hospital, à dinâmica dos horários de trabalho e/ou plantão dos profissionais solicitantes e das psicólogas, nem sempre é possível que o primeiro contato com os pacientes seja precedido pela conversa com o profissional solicitante.

"Depende... Depende bastante. (...) eles deixam o pedido e nós temos 48 horas para responder esse pedido. O que a gente tenta fazer é 
primeiro com o profissional, mas, se a gente não encontra o profissional naquele momento, a gente atende o paciente $(\ldots)^{\prime \prime}(\mathrm{P} 2)$.

As psicólogas entrevistadas ressaltaram que, quando abordam o solicitante antes do primeiro contato com o paciente, buscam informações sobre o caso clínico do paciente. Dentre essas informações estão as observações feitas pelo profissional solicitante que o direcionaram ao levantamento de hipóteses e, então, à solicitação de uma interconsulta:

"(...) eu pergunto "ah, qual foi o caso", "o que te fez perceber que precisava de uma avaliação psicológica?" (...) quais as condições clínicas dele [o paciente]. (...) então, sempre converso para agregar as informações daquele profissional, para enxergar aquele paciente da maneira mais integral possível, e não ficar só na minha visão psicológica" (P1).

Quando o primeiro contato ocorre diretamente com o paciente, as entrevistadas relataram que, antes de mais nada, oferecem ao paciente uma apresentação da profissão de psicólogo e ressaltam, brevemente, o motivo de sua presença. Em seguida, começam a coletar dados a respeito do próprio paciente, sem que seja enfatizado o motivo descrito na solicitação da interconsulta.

"Chego lá, me apresento, digo que sou psicóloga e vim conhecê-lo, (...) sou sempre bem franca com o paciente, né, e vejo como ele está (...). Vejo o suporte familiar que aquele paciente tem, (...) e quais as queixas que partem dele, porque muitas vezes a queixa é das equipes, (...) chego lá realmente para conhecer o paciente" (P1).

\section{O atendimento ao paciente}

Em suas respostas, as psicólogas entrevistadas revelaram suas preocupações em estabelecer um vínculo com o paciente. Para tanto, ressaltaram a necessidade de respeitar o ritmo do paciente e, na medida em que ele permita, coletar os dados necessários para a compreensão total da situação. Desta maneira, não permanecem focadas apenas no motivo indicado pelo profissional solicitante.

"Acho que é a coleta de dados o que caracteriza o início do atendimento psicológico (...) para depois partir para as intervenções, para orientações. Então, assim, na verdade, depende do caso, né? Então, quando o paciente é uma pessoa que está conseguindo fornecer informações claras, (...) da forma como eu acredito que as respostas do paciente vão me conduzir à informação que eu quero (...). Agora, quando a pessoa está mais debilitada, (...) aí eu tenho que ter, vamos dizer, uma flexibilidade maior para conduzir essa entrevista (...); aí o acolhimento se sobressai e (...) a coleta de dados fica em segundo plano" (P3).

Segundo as participantes, quando o paciente para o qual foi solicitado o serviço de interconsulta psicológica possui um acompanhante/familiar existem duas possibilidades de atendimento. Uma delas é realizar o atendimento com a presença do acompanhante, se o paciente assim o deseja ou está incapaz de se comunicar com a psicóloga; a outra maneira é solicitar ao acompanhante que aguarde do lado de fora do quarto, com o consentimento do paciente, e é 
proporcionado atendimento ao acompanhante/familiar após o atendimento do paciente, ainda que não disponham de um lugar adequado para esse trabalho.

"(...) O ideal, quando o paciente tem condição de se comunicar sozinho, é que a gente faça essa avaliação separadamente, depois, em um outro momento, a gente pode fazer uma avaliação conjunta, para observar a interação do paciente com o familiar. (...) Quando que eu não chegava a fazer isso: no estado de criança, paciente muito idoso, debilitado, com dificuldade de comunicação, ou então, aquele paciente que está muito assustado (...). [atendimento ao acompanhante/familiar] Na sequência, e no corredor, na maioria das vezes. $90 \%$ das vezes, no corredor. Não tem uma sala, né?" (P3).

\section{O encerramento com o paciente}

A partir dos relatos das entrevistadas, compreende-se que o encerramento do atendimento psicológico junto ao paciente pode ocorrer de dois modos. Um deles é proporcionar continuidade de atendimento aos pacientes que se mantiverem internados, assim, realiza-se um resumo do que foi abordado no atendimento e são combinados encontros subsequentes, e dá-se a disponibilidade para o paciente chamar a psicóloga quando julgar necessário. O outro modo está relacionado aos pacientes em vias de receber alta, aos quais é realizado um resumo do que foi abordado e, caso necessário, é encaminhado para o atendimento psicológico ambulatorial.

"(...) a gente vê que ele precisa de continuidade, então, na finalização (...) combino um novo, um outro atendimento. (...) quando encerra o processo de internação, e a gente percebe que o paciente está precisando (...) iniciar um tratamento psicológico em clínica, (...) entramos em contato com o Centro de Saúde de referência, para ver sobre a existência de Serviço de Psicologia e a possibilidade de encaminhar para lá (...)" (P2).

Finalização do processo de interconsulta psicológica junto ao solicitante

Segundo as participantes, a procura pelo profissional solicitante do serviço de interconsulta psicológica ao final do atendimento é necessária e importante. 0 retorno ao solicitante, após a finalização do atendimento, reassegura o tratamento do paciente e permite compreender se a solicitação feita pelo profissional foi atendida, além de proporcionar melhoras na relação paciente-equipe de saúde.

"(...) registro no prontuário e vou procurar o profissional para dar a devolutiva do que foi mais importante, se eu identifiquei alguma situação que esteja comprometendo a qualidade do tratamento. (...) eu dou no sentido de uma sugestão para a equipe médica. Na verdade, não há uma sistematização" (P3).

\section{Limitações e vicissitudes da prática}

Para as participantes, os aspectos limitantes e dificultadores para a realização da interconsulta psicológica estão relacionados à infraestrutura do hospital, à formação profissional das demais áreas da saúde, ao reconhecimento da atuação do psicólogo no contexto hospitalar e à insuficiente possibilidade de integração do psicólogo como membro da equipe de saúde. 
"Eu acho que o principal que falta é um espaço físico (...)" (P1).

"(...) em questão de ter um ramal, em ter um espaço, assim, também, físico, concretiza (...) essas duas coisas já facilitariam bastante, $(. .$. (P2).

"Boa pergunta. (...) o serviço de interconsulta funciona muito bem no hospital, acredito nisso. O ideal é que houvesse uma psicóloga, em cada unidade, aonde existe serviço de interconsulta, uma psicóloga assistencial, que pudesse fazer o seguimento dos casos" (P3).

"(...) primeiro, uma das questões, eu acho, assim, muitos dos profissionais de outras áreas não sabem muito bem por qual motivo pedir uma interconsulta, falando da Psicologia (...). Outra coisa, eu acho que muitas vezes, pelo funcionamento do hospital, a gente tem dificuldade de discutir com a equipe que pediu a interconsulta, então, isso dificulta até a nossa atuação (...)" (P4).

\section{DISCUSSÃO}

De modo geral, os resultados indicam que, como o afirmado por NogueiraMartins (1995), a interconsulta psicológica é responsável por auxiliar no diagnóstico e tratamento de pacientes com problemas psiquiátricos ou psicossociais; intermediar a relação entre paciente, equipe de saúde e familiares; facilitar a comunicação, cooperação e elaboração dos conflitos ao incluir um olhar para a subjetividade de modo a considerar todos os aspectos envolvidos no processo do adoecer. Ainda, os depoimentos das participantes indicam que, como apontado por Figueiredo (1997 citado por Rossi, 2008), a interconsulta pode ser definida como um processo de acolhimento, esclarecimento e interpretação que busca desvendar conteúdos ao considerar a subjetividade como determinante das ações dos sujeitos.

Nas entrevistas, as participantes ressaltam uma compreensão própria referente ao posicionamento profissional de como devem realizar esta prática que engloba: preocupação com a coleta de dados do paciente, buscar oferecer um cuidado integral ao mesmo, compreender a queixa e diferenciar se a mesma provém do paciente ou da equipe multidisciplinar, realizar uma intervenção adequada, ofertar atenção ao familiar presente e finalizar o atendimento junto ao paciente, no prontuário e oferecer uma devolutiva ao profissional solicitante. Este processo está de acordo com o proposto por Nogueira-Martins (1995) e Rossi (2008) citado anteriormente, devendo haver uma preocupação em realizar uma coleta e análise dos dados referentes ao ambiente e à demanda, proporcionando um diagnóstico situacional no qual são ressaltadas as reações psicológicas dos envolvidos para, então, realizar uma devolutiva completa ao profissional solicitante.

As mesmas puderam descrever, à sua maneira, o modo como realizam o atendimento a cada solicitação de interconsulta, diferenciando entre as diversas condições dos casos que são atendidos: contato primário com o profissional 
solicitante ou não, presença de familiar/acompanhante ou não, paciente em condição de comunicar por si mesmo ou não. No entanto, de maneira específica, excetuando-se pelo contato inicial com o paciente, a partir dos resultados não é possível caracterizar as etapas e respectivos procedimentos e instrumentos utilizados na interconsulta psicológica pelas profissionais que a realizam no hospital onde ocorreu o estudo.

Conforme pronunciado pela participante P3, citada na categoria "finalização do processo de interconsulta psicológica junto ao solicitante", não há uma sistematização desta prática. Com um olhar global para os dados revelados através das entrevistas, pode-se observar que não há uma sistematização do processo como um todo, pois cada etapa do atendimento dependerá das condições que serão encontradas. Acredita-se que esses achados sejam coerentes com o indicado por Ismael (2010), ou seja, apesar de haver, hoje, um maior número de profissionais na área hospitalar, ainda persiste uma série de dificuldades como a própria inserção do psicólogo na unidade institucional e a deficiência do instrumental teórico para atuação nesta área específica.

Os maiores empecilhos encontrados pela análise das próprias participantes são: a falta de contato inicial com o profissional solicitante que, por muitas vezes, pela grade de horário não coincidir impossibilita ao psicólogo buscar compreender melhor a motivação do profissional antes de atender à solicitação de interconsulta, e a dificuldade em realizar a devolutiva junto ao profissional solicitante e à equipe completa para discussão do caso e das ações seguintes. O conhecimento da própria equipe diante dos motivos considerados pertinentes para solicitar o atendimento psicológico também é um obstáculo, assim como o espaço físico para realizar os atendimentos e o número de psicólogos presentes para atender às demandas.

Devido ao fato de a Psicologia trabalhar com aspectos nem sempre fáceis de serem encarados, os psicólogos têm mais dificuldade em definir claramente seu papel do que a maioria dos outros profissionais da saúde no ambiente hospitalar. Muitas vezes, a equipe não consegue diferenciar o sofrimento psicológico do paciente do seu próprio, conforme relatado pela P1 na categoria "primeiro contato". Quando isso ocorre, o psicólogo tem um trabalho duplo: atender ao paciente e retornar à equipe para avaliar as demandas da mesma (Gazotti, 2017).

O psicólogo hospitalar busca conhecer a situação existencial e subjetiva do paciente e sua relação com a doença, de modo que a equipe passa a entender a atuação do psicólogo como um tradutor da relação médico/enfermeiro-pacientefamiliar (Saldanha, Rosa \& Cruz, 2012). Apesar das técnicas de trabalho do psicólogo ainda serem desconhecidas por parte da equipe de saúde, a mesma compreende a importância da atuação deste profissional junto ao paciente, familiares e a própria equipe (Kirchner, Granzotto \& Menegatti, 2012).

Assim, realizar uma boa prática e buscar por uma sistematização da interconsulta psicológica, é qualificar uma das principais atividades do psicólogo hospitalar, especialmente em hospital-escola (Gazotti \& Prebianchi, 2014; Rossi, 
2008). De fato, na última década, a preocupação em buscar a sistematização da prática da psicologia hospitalar aparece como um movimento crescente na comunidade acadêmica e científica que se dedica ao estudo dessa área de atuação. Estudos revelam a existência de um consenso sobre o conceito de interconsulta, porém, mostram que sua prática ainda carece de investigações com adequado delineamento metodológico e que abordem os resultados das intervenções realizadas (Andreoli \& Mari, 2002; Carvalho \& Lustosa, 2008; Rossi, 2008). A ausência de normas e diretrizes protocolares claras para esta assistência psicológica à internação hospitalar dificultam a comprovação, por níveis de evidência científica, de sua eficácia, conforme já demonstrado por Turra (2011).

Contudo, Chiattone (2014) já indicava a impossibilidade de contextualizar a psicologia hospitalar dentro de um paradigma científico, uma vez que seus pressupostos não se encontram firmemente estabelecidos. Angerami-Camom (2010 citado por Nunes \& Prebianchi, 2011) afirma que, desde a formação acadêmica do psicólogo, faltam subsídios teóricos para uma prática no contexto hospitalar, sendo essa formação muito restrita para sedimentar a prática na realidade institucional.

A análise dos empecilhos anteriormente citados, demonstra, no entanto, que a maioria das dificuldades encontram-se atreladas às características do ambiente hospitalar. Isso significa que a falta de sistematização da interconsulta psicológica ou atendimento a um protocolo, não pode ser atribuída apenas aos aspectos da formação profissional do psicólogo; mas também, como resultado das políticas e funcionamento das instituições hospitalares - as quais têm demonstrado limitações na sua capacidade de concretizar o cuidado integral e humanizado preconizado pelo modelo biopsicossocial de atenção à saúde.

\section{CONSIDERAÇÕES FINAIS}

Neste trabalho, buscamos conhecer, a partir das vivências dos profissionais psicólogos, os aspectos metodológicos e técnicos da interconsulta psicológica realizada em um hospital universitário. Acreditamos que através das descrições do contexto da pesquisa e das características dos entrevistados, os leitores poderão considerar a relevância dos achados e interpretações à sua própria situação. O que faz com que o número de participantes não seja um fator limitante.

Ao final deste estudo, através das vivências das participantes, concluímos que a interconsulta psicológica tem importância fundamental para a inserção da Psicologia no contexto do hospital geral. Esta inserção possibilita atender à necessidade de expandir o saber biopsicossocial na compreensão e cuidado da patologia, de modo a modificar as concepções habituais baseadas no modelo biomédico.

Destacam-se, todavia, limitações ou lacunas que envolvem a forma de diagnóstico e a conduta dos profissionais interconsultores, prejudicando o 
potencial de tal atividade como prática específica e legítima do psicólogo hospitalar. A não padronização da avaliação e dos critérios diagnósticos utilizados dificulta o cruzamento de informações em pesquisas científicas, de modo a interferir, também, nas intervenções realizadas. Sem uma uniformização de conduta e adequação para pesquisa, a verificação da efetividade das atuações e repercussões dos serviços prestados por interconsultores, o aperfeiçoamento e/ou desenvolvimento das técnicas e as estratégias utilizadas ficam prejudicados. Nesse sentido, julgamos imprescindíveis novos estudos sobre o tema.

\section{DECLARAÇÃO DE CONFLITOS DE INTERESSE}

Ambas as autoras, T.C.Gazotti e H.C.Prebianchi, não possuem qualquer tipo de relação com a instituição onde foi realizada a coleta de dados de modo que gere potenciais conflitos de interesse.

\section{REFERÊNCIAS}

Andreoli, P. B. A., \& Mari, J. J. (2002). Assessment of a consultation-liaison psychiatry and psychology health care program. Revista Saúde Pública, 36(2), 222-229. http://dx.doi.org/10.1590/S0034-89102002000200015.

Azevêdo, A. V. S., \& Crepaldi, M. A. (2016). A psicologia no hospital geral: aspectos históricos, conceituais e práticos. Estudos de Psicologia (Campinas), 33(4), 573-585. http://dx.doi.org/10.1590/1982-02752016000400002.

Bardin, L. (2011). Análise de conteúdo. São Paulo, SP: Edições 70.

Carvalho, M. R., \& Lustosa, M. A. (2008). Interconsulta psicológica. Revista SBPH, 11(1), 31-47. http://dx.doi.org/10.1590/S1413-73722011000200016.

Chiattone, H. B. C. (2014). A significação da psicologia no contexto hospitalar. In V. A. Angerami (Ed.), Psicologia da saúde: Um novo significado para a prática clínica (pp. 143-229). São Paulo, SP: Cengage Learning.

Gazotti, T. C. (2017). Vivências de psicólogos como integrantes de equipes multidisciplinares em hospital. (Dissertação de Mestrado). Pontifícia Universidade Católica de Campinas, Campinas, SP, Brasil. Recuperado em 29 de abril de 2019, de http://tede.bibliotecadigital.puccampinas.edu.br:8080/jspui/bitstream/tede/918/2/THAIS\%20DE\%20CAST RO\%20GAZOTTI.pdf.

Gazotti, T. C. \& Prebianchi, H. B. (2014). Caracterização da interconsulta psicológica em um hospital geral. Psicologia Teoria e Prática, 16(1), 18-30. http://dx.doi.org/0.15348/1980-6906/psicologia.v16n1p18-30. 
Giacomozzi, A. I. (2012). A inserção do psicólogo na estratégia de saúde da família e a transição de paradigma em saúde. Psico (PUCRS), 43(3), 298-308. Recuperado em 29 de abril de 2019, de http://revistaseletronicas.pucrs.br/ojs/index.php/revistapsico/article/view/7 212/8230.

Ismael, S. M. C. (2010). A inserção do psicólogo no contexto hospitalar. In S.M.C. Ismael, A prática psicológica e sua interface com as doenças. São Paulo, SP: Casa do Psicólogo.

Kirchner, L. F., Granzotto, M. D., \& Menegatti, C. L. (2012). Concepções da equipe de saúde de um hospital de Curitiba/Paraná sobre a prática de psicologia. Estudos Interdisciplinares em Psicologia, 3 (1), 24-40. http://dx.doi.org/10.1590/S1413-294X2004000200021.

Melo, C. B. (2015). História da psicologia e a inserção do psicólogo no hospital. In L. C. Santos, E. M. F. Miranda, \& E. L. Nogueira (Eds.). Psicologia, saúde e hospital: Contribuições para a prática profissional (pp. 19-32). Belo Horizonte, MG: Artesã.

Nogueira-Martins, L. A. (1995). Os beneficiários da interconsulta psiquiátrica. Boletim de Psiquiatria, 28(1), 22-23.

Nunes, G. G., \& Prebianchi, H. B. (2011). Caracterização do psicólogo em um contexto hospitalar. Anais do XVI Encontro de Iniciação Científica e I Encontro de Iniciação em Desenvolvimento Tecnológico e Inovação da PUC-Campinas, Campinas, SP.

Rossi, L. (2008). Gritos e sussurros: A interconsulta psicológica nas unidades de emergências médicas do Instituto Central do Hospital das Clínicas - FMUSP. Dissertação de Mestrado. Instituto de Psicologia, Universidade de São Paulo, São Paulo, SP. Recuperado em 29 de abril de 2019, de http://www.teses.usp.br/teses/disponiveis/47/47133/tde-12022009121121/publico/rossi me.pdf.

Saldanha, S. V., Rosa, A. B., \& Cruz, L. R. (2012). O psicólogo clínico e a equipe multidisciplinar no Hospital Santa Cruz. Revista SBPH, 16(1), 185-198. Recuperado em 29 de abril de 2019, de http://pepsic. bvsalud.org/scielo.php?script=sci arttext\&pid=S1516$08582013000100011 \& \operatorname{lng}=p t \& t \operatorname{lng}=p t$.

Santos, N. C. A., Messias, T. S., Prebianchi, H. B., Oliveira, A. E. G., \& Cardoso, C. S. (2011). Interconsulta psicológica: Demanda e assistência em hospital geral. Psicologia em Estudo, 15(2), 325-334. http://dx.doi.org/10.1590/S1413-73722011000200016.

Toro, G. V. R., Nucci, N. A. G., Toledo, T. B., Oliveira, A. E. G., \& Prebianchi, H. B. (2013). O desejo de partir: Um estudo a respeito da tentativa de suicídio. Psicologia em Revista, 19(3), 407-421. http://dx.doi.org/10.5752/P.16789563.2013V19N3P407. 
Turra, V., Costa Junior, A. L., Almeida, F. F., \& Doca, F. N. P. (2011). Contribuições da psicologia na atenção ao paciente cirúrgico: Uma análise da literatura. Comunicação em Ciências da Saúde, 22(4), 353-366.

Sobre as autoras

Thaís de Castro Gazotti é psicóloga pela Pontifícia Universidade Católica de Campinas e Mestre em Psicologia como Ciência e Profissão pela Pontifícia Universidade Católica de Campinas. Desenvolveu este trabalho enquanto bolsista de Iniciação Científica FAPIC/Reitoria. É docente na Universidade Paulista Campus Limeira, atuando nas áreas de Psicologia Clínica e da Saúde. thaisgazotti@yahoo.com

Helena Bazanelli Prebianchi é psicóloga pela Pontifícia Universidade Católica de Campinas, Mestre em Psicologia Clínica e Doutora em Psicologia como Ciência e Profissão pela Pontifícia Universidade Católica de Campinas. É docente do Programa de Pós-Graduação Stricto Sensu em Psicologia na Pontifícia Universidade Católica de Campinas, atuando nas áreas de Psicologia Clínica e da Saúde. helenabp@puc-campinas.edu.br

A autora H.B.Prebianchi desenvolveu este projeto de pesquisa a partir de sua linha de pesquisa principal, e a autora T.C.Gazotti realizou a pesquisa em campo e a redação inicial do artigo, sob supervisão e orientação de H.B.Prebianchi. H.C.Prebianchi e T.C.Gazotti são responsáveis pela redação final (revisão e edição) do artigo. H.C.Prebianchi foi responsável por cadastrar T.C.Gazotti no projeto de bolsas de iniciação científica e por requisitar apoio financeiro à FAPESP para apresentar o trabalho no congresso internacional: "20 IPLeiria Internacional Health Congress - Challenges \& Innovation in Health", School of Health Sciences of the Polytechnic Institute of Leiria (ESSLei-IPL), em Leiria, Portugal; foi também publicado em "20 IPLeiria International Health Congress - Challenges \& Innovation in Health: Abstracts", Revista Saúde Pública, 2014, 48 (n.esp).

Os autores agradecem à FAPIC/Reitoria pela bolsa de iniciação científica e à FAPESP pelo apoio financeiro para divulgação dos dados coletados em um congresso internacional. Agradecem ademais ao programa de Psicologia Hospitalar do Hospital Celso Pierro - PUC-Campinas pelo apoio na coleta de dados e à comissão de avaliação de projetos de iniciação científica da PUCCampinas pela leitura da versão inicial do texto desta pesquisa.

Recebido em: 26/07/2017

$1^{\text {a }}$ revisão em: 14/09/2017

Aceito em: 11/12/2017 